\title{
Research and Application of Harmonic Drive in Mechanical System
}

\author{
Cai Huxiang, Huang Yongmei \\ Institute of Optics and Electronics Chinese Academy of \\ Science, \\ Chengdu, 610209, China \\ chx_ioe@163.com
}

\author{
Cheng Jing \\ Chengdu Technician College, \\ Si Chuang, Cheng Du, 611731, China
}

\begin{abstract}
For reducing the effect of flexibility, backlash and friction, which is brought by the harmonic gear in the system, this paper proposes a kind of multiple loops' control method based on double speed loops. The control system includes double speed loops and current loop. Double speed loops are consist of speed loop in motor side and speed loop in load side. The speed loop in motor side is the inner loop and the speed loop in load side is the outer loop. The paper separately analyses the control performance of single speed loop and double speed loops in theory. Finally, the practical experiment results are used to validate the effectiveness of the control method based on double speed loops for inhibiting the nonlinear deadzone in the harmonic drive system.
\end{abstract} loop

Keywords-harmonic drive; flexibility; friction; double speed

\section{INTRODUCTION}

With the continuous advancement of technology, equipment gradually tends to the miniaturization trend. In the tracking control systems, its driving types mainly include direct driving of torque motor [1], reducer driving [2] and friction gear driving [3]. During these driving types, the direct driving of torque motor has always been in the lead position because of its high precision and high transmission stiffness [4].

The harmonic drive is a kind of driving mode which has been developed in the 1980s and 1990s. Because of its compact volume, large transmission ratio and light weight, it has attracted serious attentions. At the beginning, harmonic drive is mainly used in the robot field. Then it is widely applied in medical facilities, radar driving devices, aeronautical and space equipment and so on [5]. However, comparing with the direct driving of torque motor, harmonic drive has itself disadvantages, such as flexible driving and large friction [6]. Therefore, it is mainly research focus that overcomes the disadvantages of flexibility and friction in the harmonic drive [7].

To reduce the influence of nonlinear friction and low robustness in the harmonic drive, a kind of double speed loop control method is proposed to substitute the traditional single speed loop control method in this paper. And the robustness of system is analyzed in theory. In the section 5, the comparing experiment results are given. It is stated that the double speed loops control method has excellent control performance for the harmonic drive.

\section{DYNAMIC MODELS}

Harmonic drive system includes motor, harmonic drive gear, load, high precision, and control computer. The control model of the system can be found in Fig. 1.

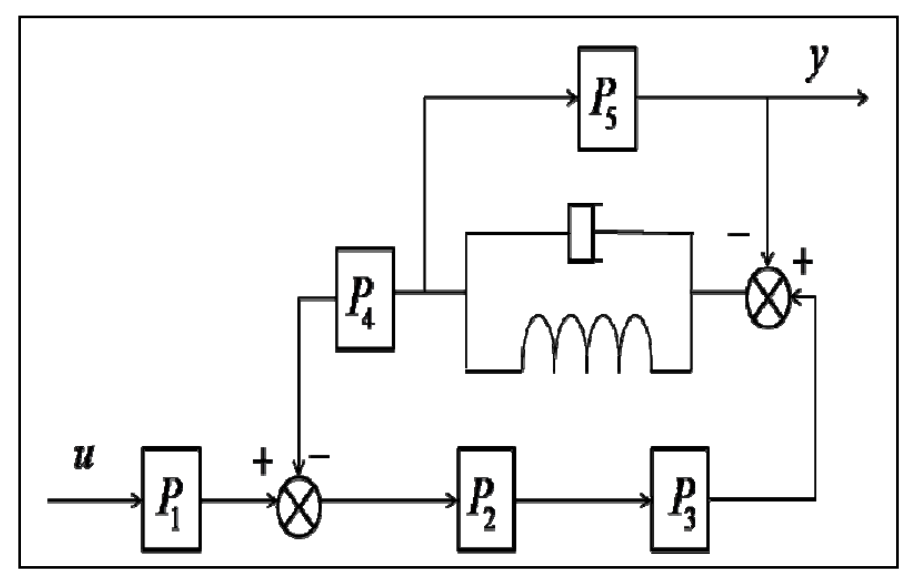

Fig. 1. The block diagram of harmonic drives system.

Where $u, y$ represent the input and output of the system, respectively; $\mathrm{P}_{1}$ is the transfer function between the input voltage and the torque; $\mathrm{P}_{2}$ is the mechanical transfer function of motor; Both $\mathrm{P}_{3}$ and $\mathrm{P}_{4}$ are the reduction gear ratio $1 / \mathrm{N}$ in the harmonic drive; $\mathrm{P}_{5}$ is the transfer function of load;

It can use the $\mathrm{K}$ to represent the stiffness coefficient of harmonic drive. The damped coefficient can be neglected. The friction $T_{f}$ will be introduced in the system. Then the system model can be predigested as follows:

As the Fig. 2 shown, the transfer function between the input voltage and output velocity can be got:

$$
y=\frac{P_{1} P_{2} P_{5} K N}{N^{2}+K P_{5} N^{2}+P_{2} K} \cdot u+\frac{P_{5} N^{2}}{N^{2}+K P_{5} N^{2}+P_{2} K} \cdot T_{f} .
$$




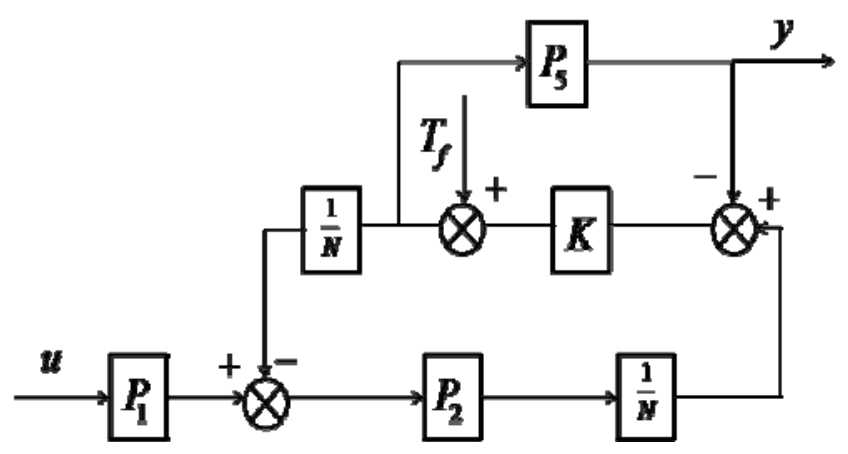

Fig. 2. The predigested block diagram of harmonic drives system.

\section{Performance Analysis of Single SpeEd Loop}

To make the system to track the given trajectory, it needs to design the closed loop controller for the system. It assumes that the speed loop controller is in the single loop system.

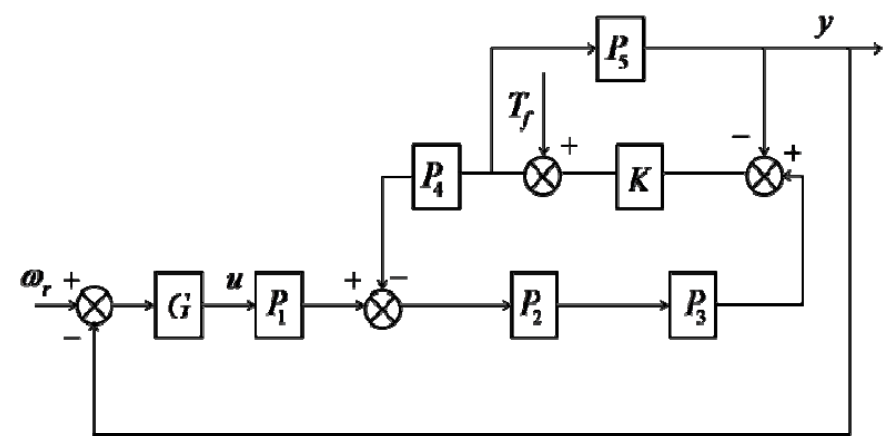

Fig. 3. The block diagram of single speed closed loop.

According to the block Fig. 3 of system, it can get the output $y$ of system:

$$
y=\frac{\frac{K}{N} P_{1} P_{2} P_{5} G \cdot \omega}{1+K P_{5}+\frac{P_{2} K}{N^{2}}+\frac{K}{N} P_{1} P_{2} P_{5} G}+\frac{P_{5} \cdot T_{f}}{1+K P_{5}+\frac{P_{2} K}{N^{2}}+\frac{K}{N} P_{1} P_{2} P_{5} G} .
$$

Generally, during designing the controller, since the reduction ratio of harmonic gear is large, the quadratic term of reduction ratio can be neglected. Then:

$$
y=\frac{\frac{K}{N} P_{1} P_{2} P_{5} G \cdot \omega}{1+K P_{5}+\frac{K}{N} P_{1} P_{2} P_{5} G}+\frac{P_{5} \cdot T_{f}}{1+K P_{5}+\frac{K}{N} P_{1} P_{2} P_{5} G} .
$$

Besides, when the controller is designed, the bandwidth needs to meet:

$$
\left|1+K P_{5}+\frac{K}{N} P_{1} P_{2} P_{5} G\right|>>1
$$

Therefore, it can get:

$$
y=\frac{\frac{1}{N} P_{1} P_{2} G \cdot \omega}{1+\frac{1}{N} P_{1} P_{2} G}+\frac{\frac{1}{K} T_{f}}{1+\frac{1}{N} P_{1} P_{2} G} .
$$

As the (5) shown, the disturbance of system is mainly restrained by the controller G(s). And the output of system also needs the controller to ensure tracking the given signal. Therefore, it must bring some difficult for adjusting the controller G(s).

On the other hand, in the (5), it can see that the stiffness coefficient of harmonic gear will have great influence on the system. Comparing with others driving, harmonic drive belongs to the flexible driving. So its stiffness coefficient $K$ will be lower than others driving. This will cause that the friction of single speed loop will have the large influence than others driving.

\section{Performance Analysis of Double Speed Loops}

To enhance the performance of system, the control method of double speed loops is introduced in the system. It assumes that the inner loop controller is $\mathrm{G}_{1}(\mathrm{~s})$ and the outer loop controller is $\mathrm{G}_{2}(\mathrm{~s})$. The control block of double speed loops is showed in Fig. 4.

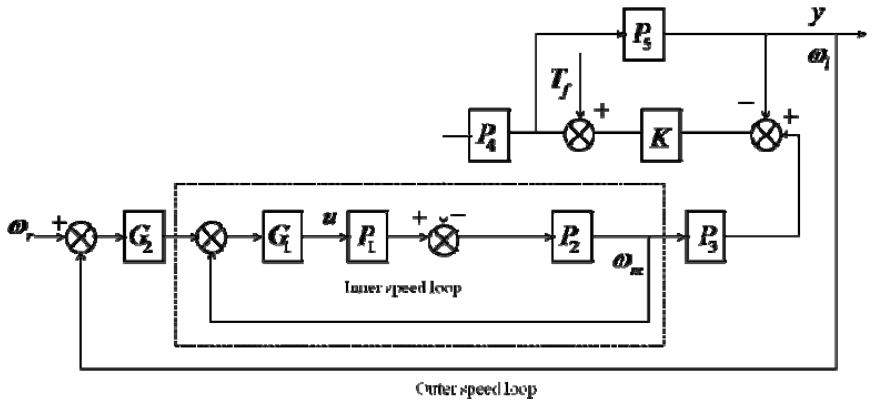

Fig. 4. The block diagram of double speed closed loops.

According to the control block of double speed loops, it can get that the output $y$ of system is,

$$
\begin{aligned}
y=\frac{\frac{K}{N} P_{1} P_{2} P_{5} G_{1} G_{2} \cdot \omega}{1+K P_{5}+\frac{P_{2} K}{N^{2}}+\frac{K}{N} P_{1} P_{2} P_{5} G_{1} G_{2}+P_{1} P_{2} G_{1}+K P_{1} P_{2} P_{5} G_{1}} \\
+\frac{\left(P_{5}+P_{1} P_{2} P_{5} G_{1}\right) T_{f}}{1+K P_{5}+\frac{P_{2} K}{N^{2}}+\frac{K}{N} P_{1} P_{2} P_{5} G_{1} G_{2}+P_{1} P_{2} G_{1}+K P_{1} P_{2} P_{5} G_{1}}
\end{aligned}
$$

Similarly, the quadratic term of reduction ratio is neglected. The bandwidth needs to meet: 


$$
\begin{gathered}
\left|1+K P_{5}+\frac{P_{2} K}{N^{2}}+\frac{K}{N} P_{1} P_{2} P_{5} G_{1} G_{2}+P_{1} P_{2} G_{1}+K P_{1} P_{2} P_{5} G_{1}\right|>>1 ; \\
\left|1+\frac{1}{N} P_{1} P_{2} G_{1} G_{2}+\frac{P_{1} P_{2} G_{1}}{K P_{5}}+P_{1} P_{2} G_{1}\right|>>1 .
\end{gathered}
$$

It can get:

$$
y=\frac{\frac{1}{N} G_{2} \cdot \omega}{\frac{1}{N} G_{2}+\frac{1}{K P_{5}}+1}+\frac{T_{f}}{\left(\frac{K}{N} G_{2}+\frac{1}{P_{5}}+K\right) P_{1} P_{2} G_{1}}+\frac{T_{f}}{\frac{K}{N} G_{2}+\frac{1}{P_{5}}+K} .
$$

In the (10), it can see that the output of system can be adjusted by the outer loop controller $\mathrm{G}_{2}(\mathrm{~s})$ and the disturbance of system can be adjusted by the inner loop controller $G_{1}(s)$. During the double speed loops, though the influence which is brought by the disturbance includes two parts, the stiffness coefficient of harmonic gear is still a large dimension. So the influence of last term will be lower than the influence in single speed loop. And the disturbance of the second term can be restrained by the controller $\mathrm{G}_{1}(\mathrm{~s})$. Therefore, by the coordination control of two controllers $G_{1}(s)$ and $G_{2}(s)$, the control precision of system can be enhanced effectively. The experiments of last section will state that the double speed loops will have a perfect performance in the harmonic drive system.

\section{EXPERIMENT RESULTS}

The high speed BLDC is selected as the driving motor. The maximum speed of the motor is 3000r $\backslash$ min. A rotary encoder which is installed in the motor side is selected as the position sensor. The reduction ratio of harmonic gear is 100. A tachometer and an optical encoder are installed in the load side, which can measure the velocity and angle position of load. To compare the control performance of two kinds of control methods, two kinds of controller are designed for the system. The given reference trajectory is $\theta=4.5 \sin (0.33 \mathrm{t})\left({ }^{\circ}\right)$.

1) Single speed loop PI controller. The angle velocity of load which is measured by the tachometer is considered as the velocity feedback signal in the controller. The angle position of load which is measured by the optical encoder is considered as the position feedback signal. Then the speed loop controller and position loop controller can be designed.

2) Double speed loops PI controller. The angle velocity of motor which is got by differencing angle position signals of motor. Besides, it can use the angle velocity of load to design another speed loop controller. Finally, it can use the angle position of load to design the load position loop controller.

The tracking results of two controllers are shown in the Fig. 5 and Fig. 6. It can see that the precision of system has a big promotion when the controller of two speed loops is applied. When the single speed loop controller is applied, the maximum error of system is about $0.132^{\prime}$. But when the double speed loops controller is applied, the maximum error of system is about $0.062^{\prime}$. The precision of system increase over one times.
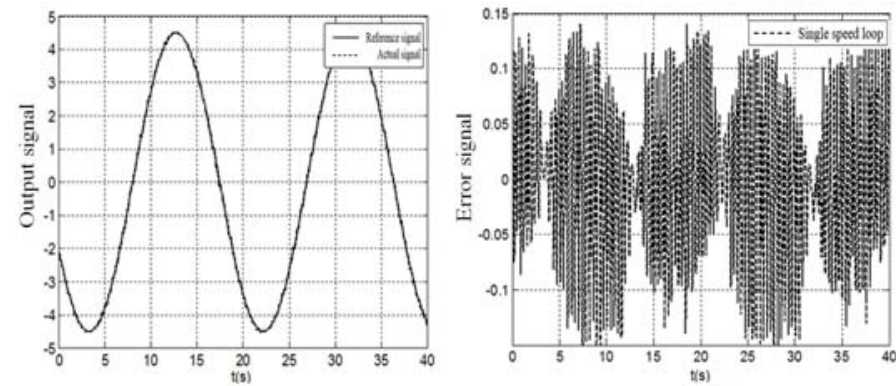

Fig. 5. The load tracking result of single speed closed loop.
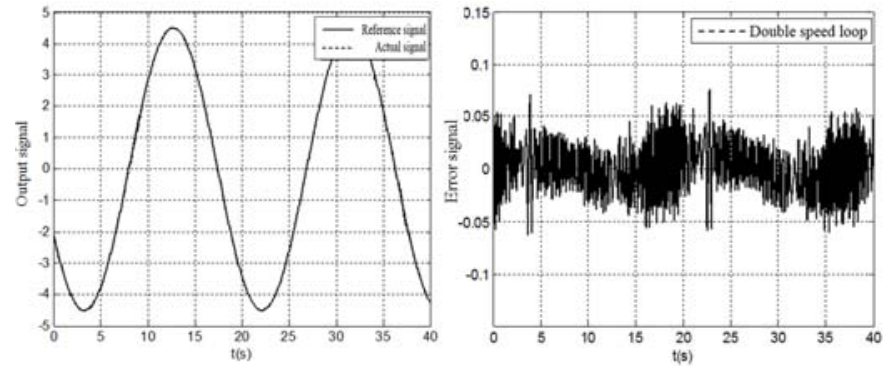

Fig. 6. The load tracking result of double speed closed loops.

\section{CONCLUSION}

To solve these nonlinear problems of flexibility, backlash and friction, a kind of multi-loops control method based on double speed loops is proposed in this paper. Besides, the performance of two control structures is analyzed in theory. Finally, by comparing with the traditional single speed loop, it is stated that the harmonic drive system will have the higher tracking precision when the double speed loops control methods is applied. The system precision increases over one times.

\section{REFERENCES}

[1] Y.Q. Yu, H. Guo, and Z.M. Xie, "Neural network adaptive sliding mode control for direct-drive servo system." T. China ElectroTech. Soc., vol. 24, no. 3, pp. 74-85, 2009.

[2] Z. Wu, and Y.D. Liu, "The practical application of harmonic drive." Aviat. Precis. Manuf. Technol., vol. 41, no. 4, pp. 23-25, 2005.

[3] G.M. Wang, Z.Q. Yao, L.S. Ma, And G.P. Li, "High precision friction drive of large telescope.” Opt. Precis. Eng., vol. 12, pp. 592-597, 2004.

[4] H. Wang, J. Cheng, L.P. Yin, and B. Miao, "Servo control technologies in electro-optical tracking system." J. Changchun U. Technol., vol. 30, no. 5, pp. 533-539, 2009.

[5] Prasanna S. Gandhi, Fathi H. Ghorbel. "Closed-loop compensation of kinematic error in harmonic drives for precision control applications." IEEE T. Control Syst. Technol., vol. 10, pp. 759-768, 2002.

[6] T. Tjahjowidodo, F. Al-Bender and H.Van Brussel. "Theoretical modeling and experimental identification of nonlinear torsional behavior in harmonic drives." Mechatronics., pp. 497-504, 2013.

[7] D. Vassileva, Y. Kiyosawa, and M. Suzuki. "Sensorless torque control a robot with harmonic drive reducers". Mech. Based Des. Struct. Mach., pp. 253-267, 2011. 